\title{
Non-monotonic temperature dependent transport in graphene grown by Chemical Vapor Deposition
}

\author{
J. Heo ${ }^{1}$, H. J. Chung ${ }^{1}$, Sung-Hoon Lee ${ }^{1}$, H. Yang ${ }^{1}$, D. H. Seo ${ }^{2}$, J. \\ K. Shin ${ }^{1}$, U-In Chung ${ }^{1}$, S. Seo ${ }^{1}$ * E. H. Hwang ${ }^{3}$, and S. Das Sarma ${ }^{3}$ \\ ${ }^{1}$ Semiconductor Devices Lab, Samsung Advanced Institute of Tech., \\ Giheung-Gu, Yongin-Si, Gyeonggi-Do 449-712, Korea \\ ${ }^{2}$ Solid State and Photonics Lab, Stanford University, Stanford, CA 94305, USA and \\ ${ }^{3}$ Condensed Matter Theory Center, Department of Physics, \\ University of Maryland, College Park, MD 20742-4111
}

\begin{abstract}
Carrier density and temperature-dependent resistivity of graphene grown by chemical vapor deposition (CVD) is investigated. We observe in low mobility CVD graphene device a generic insulating behavior at low temperatures, and eventually a metallic behavior at high temperatures, manifesting a non-monotonic temperature dependent resistivity. This feature is strongly affected by carrier density modulation with the low-density samples exhibiting insulating-like temperature dependence upto higher temperatures than the corresponding high-density samples. To explain the temperature and density dependence of the resistivity, we introduce thermal activation of charge carriers in electron-hole puddles induced by randomly distributed charged impurities. Our observed temperature evolution of resistivity is then understood from the competition among thermal activation of charge carriers, temperature-dependent screening, and phonon scattering effects. Our experimental results are in good agreement with recent theories of graphene transport.

PACS numbers: 72.80.Vp, 81.05.ue, 72.10.-d, 73.22.Pr
\end{abstract}

\section{INTRODUCTION}

Graphene, a two dimensional honeycomb lattice of carbon atoms composed of two identical interpenetrating triangular sub-lattices, has attracted research interest because of its novel linear dispersion relation resulting in unique electronic properties such as room temperature intrinsic carrier mobility $\underline{\underline{1}} \underline{\underline{4}}$. Exploiting such superior electronic properties combined with recently developed large scale growth of graphene by Chemical Vapor Deposition $(\mathrm{CVD})^{5-\underline{7}}$, has led to field effect devices integrated on a wafer scale showing promise for future electronic applications ${ }^{8}$. However, field effect performance of exfoliated graphene is adversely affected by carrier scattering by charged impurities in the surrounding media ${ }^{9} .10$, surface phonons $11-13$ on gate dielectric, and graphene acoustic phonon ${ }^{11,14}$. Although graphene grown by CVD shows outstanding uniformity and mobility on a wafer scale, field effect transport measurement performed after the transfer process, may be strongly affected by undesirable impurity scattering 15 . Therefore, understanding transport on CVD-grown graphene influenced by impurity scattering is an important issue for funamental physics and for further improvement of device performance.

In this work we are presenting an experimental study of monolayer graphene transport properties, with the emphasis on understanding the temperature dependent resistivity, complemented by a detailed comparison with theoretical calculations of graphene resistivity limited by charged impurity and phonon scattering. Such a combined experimental and theoretical study has not been undertaken for CVD graphene. Our work is closest in spirit to the early work by Tan et al $\underline{\underline{16}}$ in exfoli- ated graphene where, however, only the low-temperature density-dependent transport properties of graphene were studied in a combined experimental-theoretical investigation to establish the dominant role of charged impurity scattering in determining the low-temperature graphene electrical conductivity. Early work on graphene transport properties $\$ 2.3$ emphasized the weak temperature dependence of graphene resistivity upto the room temperature, presumably because of the weakness of the basic electron-phonon coupling in graphene. Such a weak temperature dependent conductivity is unheard of in electronic materials where phonons invariably dominate the room-temperature resistivity. In graphene, the very weak phonon effects on the electrical conductivity have only recently been experimentally observed ${ }^{17}$ verifying earlier theoretical predictions ${ }^{14}$. Although there have been several earlier experimental works on the temperature dependence of transport properties in exfoliated graphene, our current work is the only work in the literature, to the best of our knowledge, which reports on the measured temperature dependence of graphene resistivity in CVD graphene. Our work is also the only one in the literature combining experiment with detailed theory to shed light on the physical processes controlling the temperature dependence of graphene resistivity. One of our most important findings is that the low carrier density (and low temperature) temperature-dependence in graphene is dominated to a large extent by carrier activation across the potential fluctuations created by random charged impurities in the environment (which also lead to the inhomogeneous electron-hole puddle formation responsible for the graphene minimum conductivity phenomenon). We compare our experimental results with a recent theory ${ }^{18}$ on the puddle induced temperature de- 
pendence in graphene transport properties. obtaining excellent agreement. Such a comparison between theory and experiment for graphene transport at low temperature (and densities) where puddles dominate transport properties is completely new in the literature.

One other important indirect consequence of our work is the conclusion that graphene resistivity seems to manifest similar qualitative temperature and density dependence for similar mobility samples independent of how it is made, i.e. our CVD-grown samples exhibit a temperature dependent resistivity qualitatively similar to exfoliation graphene samples of similar mobility. This establishes beyond any doubt that the factor determining graphene transport properties (both density and temperature dependence) is the effective "zero-temperature" (i.e. very low-temperature) mobility which itself is determined by the charged impurity distribution in the environment. Thus, improving graphene mobility for electronic applications depends entirely on making graphene purer by removing the impurity content from the environment. Having CVD graphene helps only if it has low impurity content, which, according to our work, is no longer the case when CVD graphene is transferred.

We investigate temperature $(T)$ dependent resistivity, $\rho(T)$, of graphene grown by CVD at various carrier densities $\left(n_{g}\right)$. In low mobility samples, a strong insulating behavior at low temperature and a metallic behavior at high temperature is observed, resulting in nonmonotonicity. (We define insulating/metallic as $d \rho / d T$ being negative/positive in the sample - this is purely an operational definition which is used extensively in the literature: all our samples are metallic in reality since a mobility can be defined.) This feature persists up to relatively high carrier density. On the other hand, high mobility samples show overall metallic behavior from fairly low to high carrier density. To understand this peculiar transport property of CVD graphene, we introduce the concept of thermal activation of charge carriers in electron-hole puddles generated by charged impurity $\underline{19}$ which strongly affects the graphene mobility. Anomalous $T$ and $n_{g}$ dependence of transport is well described theoretically by the competition among thermal activation, screened impurity scattering, and phonon scattering. Thus, puddles play a very important role in controlling the temperature dependent CVD graphene transport. We compare our data with a recently developed detailed theory $\underline{\underline{18}}$.

Temperature dependence of carrier transport properties, e.g. resistivity, in electronic materials is often attributed to be arising from carrier-phonon interaction since phonons are thermally excited quantized-vibrations of the underlying lattice $\stackrel{1}{-}$. For example, the temperature dependence of electrical resistivity in normal metals like $\mathrm{Al}$ or $\mathrm{Cu}$ is essentially entirely controlled by the electron-phonon interaction strength in the metal. Such 'metallic' temperature dependence always involves an increasing resistivity with increasing temperature, i.e. $d \rho / d T>0$, and often, the definition of a metal is taken to be synonymous with the $d \rho / d T>0$ behavior (or very weak temperature dependence of resistivity) with a finite (and presumably, not too large) zero-temperature extrapolated resistivity. An insulator, on the other hand, has infinite (zero) resistivity (conductivity) at $T=0$, and increasing $T$ typically increases its conductivity since activated or variable-range hopping transport become allowed at finite temperature. An insulator or a localized system thus has $d \rho / d T<0$ with an extrapolated zerotemperature resistivity which is infinite (or very large). Thus, $d \rho / d T<0$ is often taken to be an operational definition of an insulator. This operational definition of an effective 'metal' $(d \rho / d T>0)$ versus an effective 'insulator' $(d \rho / d T<0)$ is general and powerful, and has long guided experimental and theoretical research in electronic materials (metals, semiconductors, doped materials). However, we emphasize that 'metallic' and 'insulating' used in our paper are terminologies for the temperature dependence of resistivity. ( $d \rho / d T$ being negative/positive does not necessarily indicate a $T=0$ true insulator/metal.)

Given the above very general consideration, it is therefore always very interesting and intriguing when an otherwise well-defined metallic system exhibits insulating (i.e. $\quad d \rho / d T<0)$ temperature dependence. It turns out that graphene is indeed such a rather unusual electronic material which often manifests $d \rho / d T<0$ in some carrier density and temperature range while exhibiting the expected 'metallic' behavior $d \rho / d T>0$ in other regimes of density and temperature $1,10-12,14$. In fact, for some samples (and at some specific values of carrier density) even non-monotonic temperature dependence with resistivity decreasing with $T$ first and then increasing with $T$ is observed. In the current paper, we present a detailed experimental study of the temperature dependence of graphene transport properties comparing it with a quantitative theoretical analysis to show how and why such interesting temperature dependence could arise. The non-monotonicity in the graphene resistivity indicates competing physical processes, and indeed, there are several independent contributions to graphene transport which all depend non-trivially on density and temperature. These physical processes combine to give rise to the observed temperature dependence as we show in the current work. We emphasize that although some aspects of this temperature dependence were earlier studied experimentally in the literature for exfoliated graphene on substrates and for suspended graphene $\frac{11,12,20,21}{}$, ours is the first such temperature-dependent transport study for graphene grown by the CVD technique. Ours is also the first experimental study where the measurements are carefully compared with a detailed theoty.

The competing physical mechanisms contributing to the temperature dependent graphene transport are phonons (which are known to be weak in graphene), screening, Fermi surface effects, and carrier activation across potential fluctuations associated with the density inhomogeneities or puddles in graphene which dominate 
its low-density properties. Of these four mechanisms, the first two (phonons and screening) always produce metallic behavior whereas the last two (Fermi surface and activation) always produce 'insulating' behavior. In general, both insulating effects (i.e. Fermi surface effects and activation across puddles) are stronger at low carrier densities whereas the two 'metallic' mechanisms are stronger at higher carrier densities. Thus, we expect graphene to preferentially exhibit metallic (insulating) behavior at higher (lower) carrier densities. Of course, at high enough temperature, phonons will eventually dominate, and all graphene samples should eventually exhibit metallic transport properties (i.e. $d \rho / d T>0$ ), and therefore, at some densities we expect to see nonmonotonicity in the graphene resistivity as a function of temperature. All these expectations are beautifully manifested in our experiments and we find reasonable quantitative agreement with theory. It is interesting and nontrivial, but eventually quantitatively understandable, that graphene shows a complex interplay of effective insulating and metallic behaviors superposed on each other as a function of density with the insulating (metallic) behavior dominating low (high) densities without any real localization or gapped insulator physics being present in the system. Ours is the first complete study of this phenomenon in CVD graphene with direct comparison between experiment and theory.

The paper is organized as follows. In Sec. II we describe the sample preparation and experimental results for resistivity. In Sec. III we provide the theoretical analysis of measured non-monotonic resistivity. We conclude in Sec. IV with discussion.

\section{EXPERIMENTS}

Graphene devices in this study are synthesized by Inductively Coupled Plasma enhanced Chemical Vapor Deposition (ICP-CVD) on $\mathrm{Cu}$ substrate. During growth process, the substrate is heated to $650^{\circ} \mathrm{C}$ within 10 min under $\sim 10^{-7}$ torr, then treated with $\mathrm{H}_{2}$ plasma. After purging with $\mathrm{Ar}$ for a couple of minutes, $\mathrm{C}_{2} \mathrm{H}_{2}$ is added $\left(\mathrm{C}_{2} \mathrm{H}_{2}: \quad \mathrm{Ar}=1\right.$ : 40) for graphene growth at the same temperature. For the graphene transfer, Graphene/Metal $/ \mathrm{SiO}_{2} / \mathrm{Si}$ substrate was spin-coated with PMMA (Aldrich, 950 A4) and attached a pressure sensitive adhesive ultraviolet-tape. Peeling the tape against $\mathrm{Si}$ wafer physically separates Tape/PMMA/Graphene/Metal layer due to poor adhesion of metal film and $\mathrm{SiO}_{2}$. After etching of underlying $\mathrm{Ni} / \mathrm{Cu}$ by soaking in $\mathrm{FeCl}_{3}$ and cleaning in water, Tape/PMMA/Graphene layer was pressed onto the $\mathrm{SiO}_{2}(300 \mathrm{~nm}) / \mathrm{Si}$ substrate with pre-patterned electrodes $(\mathrm{Cr} / \mathrm{Au}$ of $10 / 50 \mathrm{~nm})$. The successive removal of tape and PMMA in methanol and acetone, respectively, leaves only graphene layer over pre-patterned marks. Graphene is etched to rectangular shape by $\mathrm{O}_{2}$ plasma as shown in the inset of Fig. 1(a). The distance between source and drain electrodes is $8 \mu \mathrm{m}$ and the width of channel is $7 \mu \mathrm{m}$. The T-dependent resistivity is recorded using 4-probe measurement technique for $4 K<T<300 K$ under $\sim 10$ 5 torr after 24 hour annealing at $150{ }^{\circ} \mathrm{C}$. This annealing process before measurement gets rid of hole-doping, and moves the Dirac Point to near zero gate voltage $\left(V_{g}\right)$.

Single layer graphene was confirmed by Raman spectroscopy ${ }^{22}$ with single Lorentzian fit of $2 \mathrm{D}$ peak at $2700 \mathrm{~cm}^{-1}$ in Fig. 1(a) and optical transmittance of $97.7 \%$ at $550 \mathrm{~nm}^{23}$ in Fig. 1(b). The optical image and Raman mapping from our previous work ${ }^{15}$ verified that transferred CVD-grown graphene has no sign of wrinkles or bi, tri-layers of graphene. According to Matthiessens rule, we assume the conductivity is the sum of contributions from long-range scatterers and short range scatterers or residual resistivity. Hole mobility is then extracted using the following formula,

$\sigma\left(V_{g}\right)^{-1}=-\left[\mu_{h} C_{g}\left(V_{g}-V_{\text {Dirac }}\right)\right]^{-1}+\sigma_{\text {res }, h}^{-1} \quad V_{g}<V_{\text {Dirac }}$,

where $\sigma\left(V_{g}\right)$ is conductivity, $\mu_{h}$ is hole mobility, $C_{g}$ is capacitance of gate dielectric, $V_{\text {drag }}$ is the gate voltage at Dirac point, and $\sigma_{r e s, h}$ is residual conductivity. The measured hole mobility in a specific graphene sample in the same 6 -inch wafer has a large variation by an order of magnitude. We speculate this large variation is coming from non-uniform transfer procedure which introduces local charge impurities differently. Two groups of devices with mobility of $1,000 \sim 3,000 \mathrm{~cm}^{2} / \mathrm{Vs}$ (A) and $10,000 \sim 13,000 \mathrm{~cm}^{2} / \mathrm{Vs}(\mathrm{B})$ respectively were selected to understand the relationship between $\rho(T)$ at various $n_{g}$ and the level of disorder (high in $\mathrm{A}$, low in $\left.\mathrm{B}\right)^{9,10}$. Fig. 1(c) and (d) show $V_{g}$-dependent resistivity $\left[\rho\left(V_{g}\right)\right]$ of A and B devices, respectively. No marked difference is observed in Raman spectroscopy of these two disparate groups despite the order of magnitude variation in the mobility supporting introduction of non-uniform charge impurities during transfer. In addition, we observe a relatively weak D-peak, which indicates minimum level of structural defects in our samples.

T-dependence of resistivity $[\rho(T)]$ with reference to $\rho(4.2 \mathrm{~K})$ at carrier densities near charge neutrality point is plotted in Fig. 2(a) and (b) for A and B respectively. $\Delta V_{g}$ is equal to $V_{g}-V_{\text {Dirac }}$. The characteristic observed feature of carrier transport in our low mobility sample A (Fig. 2(a)) is a strong insulating behavior (i.e., $d \rho(T) / d T<0)$ at low temperatures $(T<200 K)$ for all densities (up to $7.2 \times 10^{11} \mathrm{~cm}^{-2}$ ). For $\Delta V_{g}>4 \mathrm{~V}$ $\left(n_{g}>2.9 \times 10^{11} \mathrm{~cm}^{-2}\right)$ a non-monotonicity in temperature dependent resistivity develops (i.e. as $\mathrm{T}$ increases, $\rho$ initially decreases and then increases forming a local minimum). The observed insulating behavior and nonmonotonicity at high density in low mobility samples is not observed in high mobility samples B (Fig. 2(b)), where the measured resistivity shows a metallic temperature dependence at higher densities even though the experimental conductivity manifests an insulating behavior at very low carrier density (up to $n_{g} \sim 2.0 \times 10^{11} \mathrm{~cm}^{-2}$ ). Interestingly, negative $d \rho(T) / d T$ at Dirac point is $\sim$ 

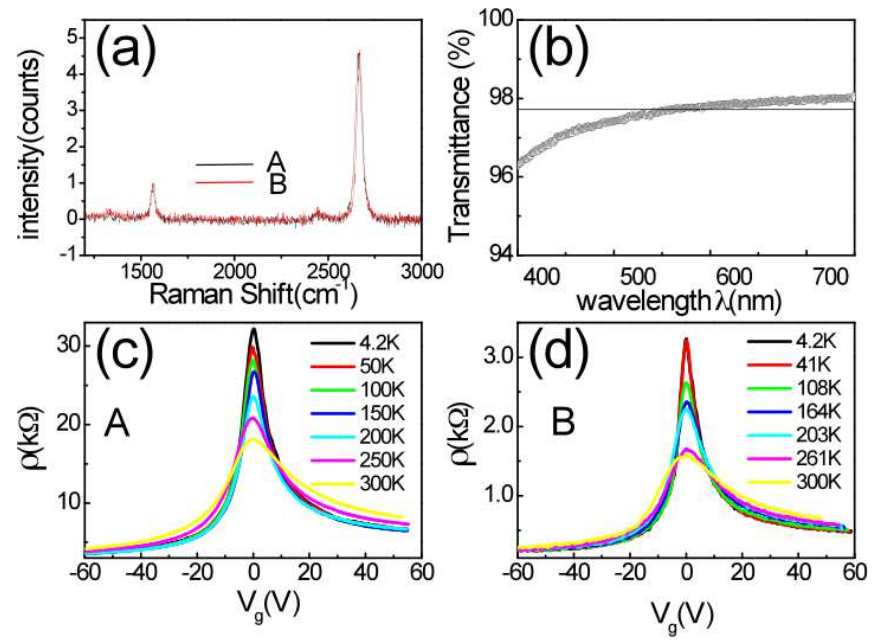

FIG. 1. (color online) (a) Raman spectroscopy on a device, inset: Optical microscope image of a device. (b) Light transmittance through CVD grown single layer graphene/Glass. (c) and (d) resistivity vs. gate voltage at various temperature for the device group of $\mathrm{A}$ and $\mathrm{B}$, respectively.

$40 \%$ for both samples independent of sample mobility, which also has been reported in high mobility suspended graphene $e^{20,24}$. Recently, ballistic transport was invoked in order to explain the strong T-dependent $\rho(T)$ at Dirac point as well as low $n_{g}$ regime 25 . However, this approach is not applicable in our low mobility devices which are obviously in the diffusive regime. The temperature dependence in our diffusive samples is presumably connected with extrinsic effects such as charged impurity scattering, which is considered the dominant scattering process for transport at low $n_{g}$. Note that only metallic behavior has been seen in graphene samples at high densities.

Another important aspect of temperature dependent resistivity is that the initial insulating behavior at Dirac point is strongly affected by carrier density modulation. Starting from the charge neutrality point, the insulating behavior becomes non-monotonic near $\Delta V_{g} \sim 4 V\left(n_{g}=\right.$ $2.9 \times 10^{11} \mathrm{~cm}^{-2}$ ) for sample A due to the coexistence of low $\mathrm{T}$ insulating and high $\mathrm{T}$ metallic behavior (Fig. $2(\mathrm{a})$ ), but for sample B, a complete transition to metallic behavior occurs already around $n_{g}=2 \times 10^{11} \mathrm{~cm}^{-2}$ (Fig. $2(\mathrm{~b})$ ).

\section{THEORETICAL ANALYSIS}

To understand $\mathrm{T}$ and $n_{g}$ dependence of different mobility devices, we consider the quantitative theory for carrier transport in the presence of electronhole puddles due to the spatially fluctuating inhomogeneous potential induced by random charged impurities. The formation of electron-hole puddles has been proposed theoretically 26 and observed in scanning tunneling spectroscopy 27 and scanning single electron transistor microscopy experiments ${ }^{28}$. The presence of electron-hole
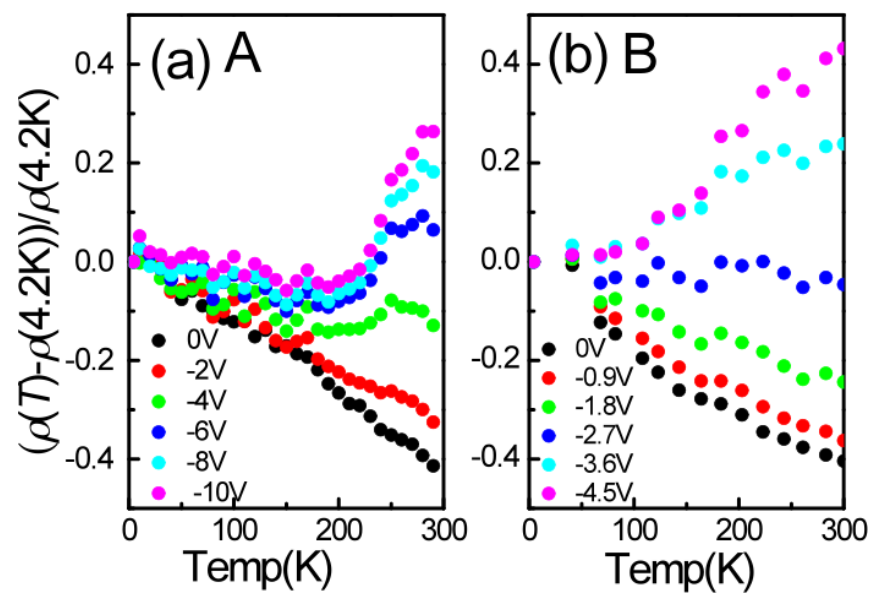

FIG. 2. (color online) $[\rho(T)-\rho(4.2 K)] / \rho(4.2 K)$ at varied $\Delta V_{g}$. (a) device A, (b) device B.

puddles was recently introduced to explain the insulating behavior in metallic bilayer graphene ${ }^{19}$. It is crucial to include the role of electron-hole puddles to explain the measured insulating behavior in our monolayer graphene samples. We incorporate the puddles into the potential fluctuations which is assumed to be described by a statistical distribution function of a Gaussian form with a standard deviation $s \stackrel{19}{\underline{19}}$ Thus, we calculate theoretically the carrier transport for our graphene samples by taking into account the highly inhomogeneous density and potential landscape. We also include the scattering by screened charged impurities, longitudinal acoustic phonon scattering and surface optical phonon scattering. Then the total scattering time is calculated by

$$
\frac{1}{\tau_{\text {tot }}}=\frac{1}{\tau_{\text {imp }}}+\frac{1}{\tau_{a p}}+\frac{1}{\tau_{s p}}
$$

where $\tau_{i m p}, \tau_{a p}$, and $\tau_{s p}$ are the transport scattering times of screened charged impurity 29 , longitudinal acoustic phonon $\stackrel{14}{ }$, and surface optical phonon $\frac{13}{3}$, respectively. The scattering time due to charged impurity $\tau_{i m p}$ is given by

$$
\begin{array}{r}
\frac{1}{\tau_{i m p}(\varepsilon)}=\frac{2 \pi n_{i}}{\hbar} \int \frac{d^{2} k^{\prime}}{(2 \pi)^{2}}\left|\frac{v_{i}(q)}{\epsilon(q)}\right|^{2} g\left(\theta_{\mathbf{k k}^{\prime}}\right) \\
\times\left[1-\cos \theta_{\mathbf{k k}^{\prime}}\right] \delta\left(\varepsilon_{\mathbf{k}}-\varepsilon_{\mathbf{k}^{\prime}}\right),
\end{array}
$$

where $n_{i}$ is the charged impurity density, $\varepsilon_{\mathbf{k}}=\hbar v_{F} \mathbf{k}$ is the carrier energy with the Fermi velocity $v_{F}$ and $2 \mathrm{D}$ wave vector $\mathbf{k}, g\left(\theta_{\mathbf{k}, \mathbf{k}^{\prime}}\right)=[1+\cos (\theta)] / 2$ is a wave function form factor associated with the chiral matrix of graphene, $\theta_{\mathbf{k k}^{\prime}}$ is the scattering angle between $\mathbf{k}$ and $\mathbf{k}^{\prime}$, $v_{i}(q)=2 \pi e^{2} / \kappa q$ is the $2 \mathrm{D}$ Coulomb potential, and $\epsilon(q)$ is the finite temperature dielectric screening function. The scattering time due to acoustic phonon mode $\tau_{a c}$ is given by

$$
\frac{1}{\tau_{a c}(\varepsilon)}=\sum_{\mathbf{k}^{\prime}}\left(1-\cos \theta_{\mathbf{k k}^{\prime}}\right) W_{\mathbf{k} \mathbf{k}^{\prime}} \frac{1-f\left(\varepsilon^{\prime}\right)}{1-f(\varepsilon)}
$$


where $f(\varepsilon)=\left[e^{(\varepsilon-\mu) / k_{B} T}+1\right]^{-1}$ is the Fermi distribution function with the chemical potential $\mu$, and $W_{\mathbf{k k}^{\prime}}$ is the transition probability from the state with momentum $\mathbf{k}$ to $\mathbf{k}^{\prime}$ state and given by

$$
\begin{aligned}
W_{\mathbf{k k}^{\prime}}=\frac{2 \pi}{\hbar} \sum_{\mathbf{q}}|C(\mathbf{q})|^{2} & \times\left[N_{q} \delta\left(\varepsilon-\varepsilon^{\prime}+\omega_{\mathbf{q}}\right)\right. \\
+ & \left.\left(N_{q}+1\right) \delta\left(\varepsilon-\varepsilon^{\prime}-\omega_{\mathbf{q}}\right)\right],
\end{aligned}
$$

where $C(\mathbf{q})$ is the matrix element for scattering by acoustic phonon, $\omega_{\mathbf{q}}=v_{p h} \mathbf{q}$ is the acoustic phonon frequency with $v_{p h}$ being the phonon velocity, and $N_{q}$ the phonon occupation number $N_{q}=1 /\left(\exp \left(\beta \omega_{\mathbf{q}}\right)-1\right)$. The first (second) term is Eq. (5) corresponds to the absorption (emission) of an acoustic phonon of wave vector $\mathbf{q}=\mathbf{k}-\mathbf{k}^{\prime}$. The matrix element $C(\mathbf{q})$ for the deformation potential is given by

$$
|C(\mathbf{q})|^{2}=\frac{D^{2} \hbar q}{2 A \rho_{m} v_{p h}}\left[1-\left(\frac{q}{2 k}\right)^{2}\right],
$$

where $D$ is the deformation potential coupling constant, $\rho_{m}$ is the graphene mass density, and $A$ is the area of the sample. The scattering time due to the surface optical phonons is given by
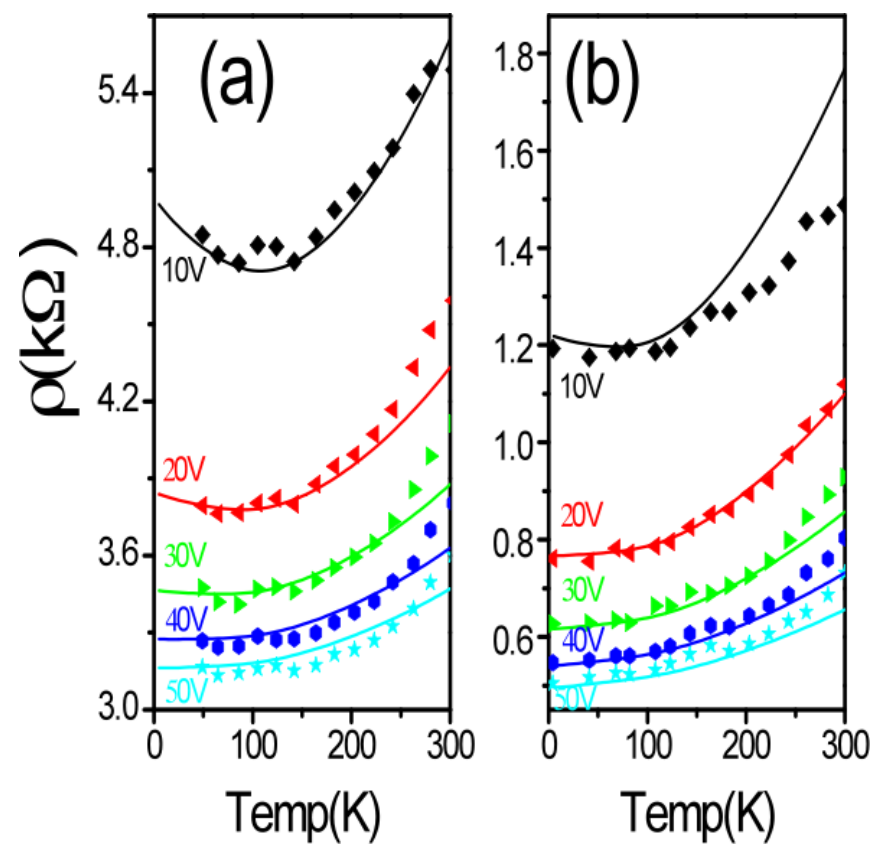

FIG. 3. (color online) $\rho(T)$ at varied $\Delta V_{g}$. Scatters are experimental data and lines are calculation for (a) device A, (b) device B.

$$
\begin{aligned}
\frac{1}{\tau_{s p}(\varepsilon)} & =\sum_{l \mathbf{k}^{\prime}} M_{0 l}^{2}\left(\mathbf{k}-\mathbf{k}^{\prime}\right) g\left(\theta_{\mathbf{k}, \mathbf{k}^{\prime}}\right)\left[\left(N_{0}+f(\varepsilon)\right) \delta\left(\varepsilon+\omega_{S O l}-\varepsilon_{\mathbf{k}^{\prime}}\right)\right. \\
& \left.+\left(N_{0}+1-f(\varepsilon)\right) \delta\left(\varepsilon-\omega_{S O l}-\varepsilon_{\mathbf{k}^{\prime}}\right)\right]
\end{aligned}
$$

absorb or emit phonons. We find that the surface phonon (7s)cattering plays an important role for $T>200 \mathrm{~K}$ and the scattering rate strongly depends on the distance between the $2 \mathrm{D}$ graphene layer and the substrate.

where the matrix elements of a surface optical phonon is given by

$$
\left[M_{0 l}(q)\right]^{2}=\frac{2 \pi e^{2}}{q \epsilon(q)} e^{-2 q d} \frac{\omega_{S O l}}{2}\left[\frac{1}{\epsilon_{\infty}+1}-\frac{1}{\epsilon_{0}+1}\right],
$$

where $d$ is the separation distance between graphene layer and substrate, $\epsilon_{0}\left(\epsilon_{\infty}\right)$ is the static (high frequency) dielectric constant, and $\omega_{S O l}$ is the $l$-th kind of surface optical phonon frequency. Then by averaging over energy we have the conductivity as

$$
\sigma(T)=\frac{e^{2}}{2} \int d \varepsilon D(\varepsilon) v_{F}^{2} \tau_{t o t}(\varepsilon, T)\left(-\frac{\partial f(\varepsilon)}{\partial \varepsilon}\right),
$$

where $D(\varepsilon)=2 \varepsilon / \pi\left(\hbar v_{F}\right)^{2}$ is the density of states of graphene.

We find that the screened charge impurity scattering dominates over phonon scattering at low $\mathrm{T}$ and strongly contributes to $\rho(T)$ for all T. The acoustic phonon scattering gives rise to a linear $\mathrm{T}$ dependent resistivity and its contribution to $\rho(T)$ is a quantitatively small even at room temperature. It is only important for high mobility and high density samples because it is independent of $n_{g}$ and sample quality. It is known that the carriers in graphene are strongly coupled to the surface optical phonons of a polar substrate such as $\mathrm{SiO}_{2}$. Consequently, the surface phonon scattering gives a large contribution to $\rho(T)$ when the carrier temperature is high enough to

We compare experimental and theoretical $\rho(T)$ in Fig. 3 incorporating all effects we discussed above. We use the charged impurity densities $\left(n_{i}\right)$ and the standard deviation of potential fluctuation $(\mathrm{s})$, (a) $n_{i}=1.8 \times 10^{12} \mathrm{~cm}^{2}$ and $s=70 \mathrm{meV}$, (b) $n_{i}=4.5 \times 10^{11} \mathrm{~cm}^{2}$ and $s=110$ $\mathrm{meV}$. To get the charge impurity densities we first fit our data at low temperature and high density limits because in these limits all phonon scattering effects are exponetionally suppressed and scattering by charged impurity dominates. For acoustic phonon scattering we use the phonon velocity $v_{p h}=2 \times 10^{6} \mathrm{~cm} / \mathrm{s}$ and the deformation potential coupling constant $D=19 \mathrm{eV} \underline{\underline{14}}$ For surface optical phonon scattering we use the two optical phonons in our calculation with $\omega_{s p 1}=63 \mathrm{meV}$ and and $\omega_{s p 2}=116$ $\mathrm{meV} \stackrel{12}{2}$ The overall resistivity scale depends on the impurity density or mobility value, but the qualitative trends in $\rho(T)$ arise from the basic aspects of the underlying scattering mechanisms. The rise in $\rho$ with increasing $\mathrm{T}$ at high temperatures is a direct result of the thermal weakening of screening and phonon effects. The decrease in $\rho$ with temperature at low temperatures arises from thermal activation in the presence of electron-hole puddles $\frac{19}{}$. The non-monotonicity of $\rho(T)$ at the intermediate temperatures arises from the competition among activation, temperature dependent screening and phonon scattering. The activated insulating behavior competes with the metallic behavior induced by the temperature 
dependent screening and phonon effects. When the potential fluctuation is smaller than the chemical potential the activation behavior is suppressed. As a result the total conductivity manifests a metallic behavior. However, for large potential fluctuations the temperature dependence by activation overwhelms the temperature dependence due to all other mechanisms and the system shows insulating behavior. In our calculations we neglect Anderson localization. But we cannot rule out that in the lowest mobility sample localization may play a role in the insulating behavior. Detailed quantitative understanding in this regime would require a more sophisticated theory which includes higher order electron-electron interactions and disorder induced localization corrections, but our theoretical results $13,14,19,26,29$ give an excellent qualitative description of the data. We also measure the resistivity (not shown) for very low mobility sample $(\mu=300$ $\mathrm{cm}^{2} / \mathrm{Vs}$ ) showing a stronger insulating behavior at low temperatures than high mobility samples of Fig. 3(a) and (b).

\section{DISCUSSION AND CONCLUSION}

In conclusion, temperature dependent transport of CVD grown graphene is investigated. For low mobility samples, an anomalous insulating behavior at low temperatures and non-monotonic at higher temperatures are observed, in contrast to the monotonic temperature dependent transport of high mobility exfoliation graphene. However, we note that a strong insulating behavior can be obsered at low mobility exfoliated graphene. Most important factor in observing an insulating temperature dependent transport is the strength of potential fluctuations which are proportional to the charged impurity density or inversely proportional to the mobility. If the sample mobility is low enough the insulating behavior is notable for even exfoliation graphene samples ${ }^{21}$. These are explained based on the transport theory incorporating thermally activated transport of inhomogeneous electron-hole puddles. Our results imply that carrier transport in CVD graphene devices, which are vulnerable to the impurities or charged defects during the transfer process, is strongly affected by underlying environments, which gives rise to lower mobility in CVD graphene than the exfoliated graphene since both CVD and exfoliated graphene use the same substrate. To get higher mobility CVD graphene a careful transfer process is required in order to keep the background impurity density low.

We have shown, through experimental measurements and detailed comparison with theoretical calculations, that the temperature dependence of graphene resistivity can be understood as arising from a complex interplay among the physical mechanisms of phonon scattering, screening, Fermi surface averaging, and activation across potential barriers in inhomogeneous puddles. The first (last) two mechanisms lead to metallic (insulating) temperature dependence with resistivity increasing (decreasing) with temperature, and therefore, the net effect could be either an insulating or a metallic temperature dependence or a non-monotonic combination of both, depending on the details of sample mobility, density, and the temperature regime. With increasing (decreasing) density and mobility, puddle effects are suppressed (enhanced), leading to stronger metallicity. With increasing temperature, phonon effects eventually dominate, so at the highest temperature (and not too low densities), the system always manifests metallic behavior with increasing resistivity with increasing temperature. At the Dirac point, activation in puddles dominate and the insulating behavior is generic. For intermediate temperatures and densities, the generic behavior of resistivity as a function of temperature is non-monotonic, as predicted in ref. 19, with the resistivity decreasing with increasing temperature at low temperatures and then eventually increasing with temperature (except at the lowest densities where puddles are dominant even at room temperatures) at higher temperatures. The crossover temperature for the transition from insulating to metallic transport behavior increases with decreasing mobility and/or carrier density. All of these observed effects are completely consistent with the predictions of the theory developed recently $\underline{18,19}$.

\section{ACKNOWLEDGMENT}

The work at the University of Maryland is supported by NRI-SWAN and US-ONR.
* sunaeseo@samsung.com

1 S. Das Sarma, S. Adam, E. H. Hwang, and E. Rossi, Rev. Mod. Phys.83, 407 (2011).

${ }^{2}$ K. S. Novoselov, A. K. Geim, S. V. Morozov, D. Jiang, Y. Zhang, S. V. Dubonos, I. V. Grigorieva, and A. A. Firsov, Science 306, 666 (2004).

3 A. K. Geim and K. S. Novoselov, Nat. Mater. 6, 183 (2007).

${ }^{4}$ K. I. Bolotin, K. J. Sikes, Z. Jiang, G. Fundenberg, J. Hone, P. Kim, and H. L. Stormer, Solid State Commun. 146, 351 (2008).
${ }^{5}$ A. Reina, X. Jia, J. Ho, D. Nezich, H. Son, V. Bulovic, M. S. Dresselhaus, and J. Kong, Nano Lett. 9, 30 (2009).

${ }^{6}$ K. S. Kim, Y. Zhao, H. Jang, S. Y. Lee, J. M. Kim, K. S. Kim, J. Ahn, P. Kim, J. Choi, and B. H. Hong, Nature 457, 706 (2009).

7 X. Li, W. Cai, J. An, S. Kim, J. Nah, D. Yang, R. Piner, A. Velamakanni, I. Jung, E. Tutuc, S. K. Banerjee, L. Colombo, and R. S. Ruoff, Science 324, 1312 (2009).

${ }^{8}$ Y. Lee, S. Bae, H. Jang, S. Jang, S. Zhu, S. H. Sim, Y. I. Song, B. H. Hong, and J. Ahn, Nano Lett. 10, 490 (2010). 
9 S. Adam, E. H. Hwang, V. M. Galitski, and S. Das Sarma, PNAS 104, 18392 (2007).

10 J.-H. Chen, C. Jang, S. Adam, M. S. Fuhrer, E. D. Williams, and M. Ishigami, Nature Phys. 4, 377 (2008).

11 J.-H. Chen, C. Jang, S. Xiao, M. Ishigami, and M. S. Fuhrer, Nature Nanotech. 3, 206 (2008).

12 K. Zou, X. Hong, D. Keefer, and J. Zhu, Phys. Rev. Lett. 105, 126601 (2010).

13 S. Fratini and F. Guinea, Phys. Rev. B 77, 195415 (2008).

14 E. H. Hwang and S. Das Sarma, Phys. Rev. B 77, 115449 (2008).

15 J. Lee, H. J. Chung, J. Lee, H. Shin, J. Heo, H. Yang, S.H. Lee, S. Seo, J. Shin, U-In Chung, I. Yoo, and K. Kim, IEDM (in press).

${ }^{16}$ Y.-W. Tan, Y. Zhang, K. Bolotin, Y. Zhao, S. Adam, E. H. Hwang, S. Das Sarma, H. L. Stormer, and P. Kim, Phys. Rev. Lett. 99, 246803 (2007).

17 Dmitri K. Efetov and Philip Kim, Phys. Rev. Lett. 105, 256805 (2010).

18 Q. Li, E. H. Hwang, and S. Das Sarma, arXiv:1105.1771

19 E. H. Hwang and S. Das Sarma, Phys. Rev. B 82, 081409 (2010).
${ }^{20}$ K. I. Bolotin, K. J. Sikes, J. Hone, H. L. Stormer, and P. Kim, Phys. Rev. Lett. 101, 096802 (2008).

21 W. Zhu, V. Perebeinos, M. Freitag, and P. Avouris, Phys. Rev. B 80, 235402 (2009).

22 A. C. Ferrari, J. C. Meyer, V. Scardaci, C. Casiraghi, M. Lazzeri, F. Mauri, S. Piscanec, D. Jiang, K. S. Novoselov, S. Roth, and A. K. Geim, Phys. Rev. Lett. 97, 187401 (2006).

23 R. R. Nair, P. Blake, A. N. Grigorenko, K. S. Novoselov, T. J. Booth, T. Stauber, N. M. R. Peres, and A. K. Geim, Science 320, 1308 (2008).

${ }^{24}$ X. Du, I. Skachko, A. Barker, and E. Y. Andrei, Nature Nanotech. 3, 491 (2008).

25 M. Müller, M. Bräuninger, and B. Trauzettel, Phys. Rev. Lett. 103, 196801 (2009).

26 E. H. Hwang, S. Adam, and S. Das Sarma, Phys. Rev. Lett. 98, 186806 (2007).

27 Y. Zhang, V. W. Brar, C. Girit, A. Zettl, and M. F. Crommie, Nature Phys. 5, 722 (2009).

28 J. Martin, N. Akerman, G. Ulbricht, T. Lohmann, J. H. Smet, K. von Klitzing, and A. Yacoby, Nature Phys. 4, 144 (2008).

29 E. H. Hwang and S. Das Sarma, Phys. Rev. B 79, 165404 (2009). 